\title{
In vitro and in silico investigation of water-soluble fullerenol
}

\section{$\mathrm{C}_{60}(\mathrm{OH})_{24}$ : bioactivity and biocompatibility}

\section{Supporting Information}

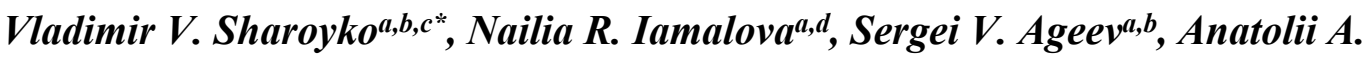
Meshcheriakov ${ }^{a, b}$, Gleb O. Iurev ${ }^{a, e}$, Andrey V. Petrov ${ }^{b}$, Dmitry A. Nerukhf, Vladimir S.

Farafonov", Lubov V. Vasina ${ }^{a}$, Anastasia V. Penkova ${ }^{b}$,Konstantin N. Semenov ${ }^{a, b, c^{*}}$

${ }^{a}$ Pavlov First Saint Petersburg State Medical University, 6-8 L'va Tolstogo ulitsa, Saint Petersburg, 197022, Russia

${ }^{b}$ Institute of Chemistry, Saint Petersburg State University, 26 Universitetskii prospekt, Saint Petersburg, 198504, Russia

${ }^{c}$ A. M. Granov Russian Research Centre for Radiology and Surgical Technologies, 70 Leningradskaya Ulitsa, Saint Petersburg, 197758, Russia

${ }^{d}$ Agrophysical Research Institute, 14 Grazhdanskii prospect, Saint Petersburg 195220, Russia

${ }^{e}$ Almazov National Medical Research Centre, 2 Akkuratova ulitsa, Saint Petersburg, 197341, Russia

${ }^{f}$ Department of Mathematics, Aston University, Birmingham, B4 7ET, UK

$g$ V. N. Karazin Kharkiv National University, 4 Svobody ploshchad', Kharkiv, 61022, Ukraine

*Corresponding author. E-mail address: knsemenov@gmail.com (K. N. Semenov), sharoyko@gmail.com (V.V. Sharoyko). 
Table S1. Hill coordinates for $\mathrm{C}_{60}(\mathrm{OH})_{24}$ binding to HSA at $298.15 \mathrm{~K}$ in the absence and presence of site markers.

\begin{tabular}{lcccc}
\hline & & \multicolumn{2}{c}{$\lg \left[\left(F_{0}-F\right) / F\right]$} & \\
\cline { 2 - 5 } $\lg Q$ & No markers & Warfarin & Ibuprofen & Digitoxin \\
\hline-6.52 & $-1.26 \pm 0.07$ & $-1.10 \pm 0.16$ & $-1.15 \pm 0.04$ & $-1.20 \pm 0.06$ \\
-6.22 & $-0.93 \pm 0.04$ & $-0.83 \pm 0.12$ & $-0.85 \pm 0.04$ & $-0.88 \pm 0.04$ \\
-6.05 & $-0.75 \pm 0.04$ & $-0.66 \pm 0.09$ & $-0.66 \pm 0.02$ & $-0.74 \pm 0.03$ \\
-5.92 & $-0.63 \pm 0.03$ & $-0.53 \pm 0.08$ & $-0.56 \pm 0.02$ & $-0.65 \pm 0.03$ \\
-5.82 & $-0.55 \pm 0.03$ & $-0.42 \pm 0.06$ & $-0.49 \pm 0.02$ & $-0.58 \pm 0.03$ \\
-5.22 & $0.03 \pm 0.01$ & $0.29 \pm 0.03$ & $0.33 \pm 0.02$ & $0.18 \pm 0.01$ \\
-5.05 & $0.32 \pm 0.03$ & $0.42 \pm 0.04$ & $0.58 \pm 0.09$ & $0.52 \pm 0.03$ \\
-4.92 & $0.56 \pm 0.04$ & $0.72 \pm 0.13$ & $0.57 \pm 0.07$ & $0.70 \pm 0.04$ \\
-4.82 & $0.63 \pm 0.03$ & $0.90 \pm 0.12$ & $0.62 \pm 0.04$ & $0.79 \pm 0.03$ \\
-4.74 & $0.78 \pm 0.07$ & $1.00 \pm 0.20$ & $0.85 \pm 0.04$ & $0.85 \pm 0.06$ \\
-4.68 & $0.85 \pm 0.08$ & $1.10 \pm 0.18$ & $0.99 \pm 0.09$ & $0.96 \pm 0.08$ \\
-4.62 & $1.04 \pm 0.08$ & $1.19 \pm 0.18$ & $1.00 \pm 0.04$ & $1.09 \pm 0.10$ \\
\hline
\end{tabular}

